\title{
De l'élasticité linéaire au cœfficient de réaction : théories, observations et ordres de grandeur
}

P. SCHMITT

Soletanche Bachy

6 , rue Watford 92000 Nanterre

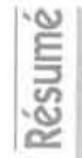

Cet article propose une comparaison systématique entre les résultats déduits de la théorie de l'élasticité linéaire et l'approche proposée par Ménard pour évaluer les cœefficients de réaction du sol vis-à-vis des fondations superficielles et des écrans de soutènement.

Ces approches sont confrontées à des mesures effectuées sur ouvrage réels, et débouchent sur une formulation générale permettant de tenir compte du comportement spécifique de chaque catégorie d'ouvrages.

\section{From elasticity to subgrade modulus : theoretical and experimental orders of magnitude}

This paper compares results derived from the theory of elasticity with Ménard's analysis of coefficients of subgrade reaction for shallow foundations and retaining strutures.

Theoretical results are then compared with site measurements, which finally results in a general relationship, taking into account specific behaviours of geotechnical structures. 


\section{Introduction}

Le coefficient de réaction est une notion empirique qui a été intensément utilisée au cours des vingt-cinq dernières années dans des domaines aussi divers que le calcul des fondations, celui des soutènements, et mème celui des tunnels.

Bien qu'intellectuellement peu satisfaisante comparativement à des méthodes numériques plus rigoureuses, cette approche continue à faire partie de la pratique quotidienne des bureaux d'études, ce que l'on peut expliquer par des considérations suivantes :

- le coefficient de réaction est une notion pratique, qui donne lieu à des programmes de calcul simples d'utilisation et bien adaptés à leur usage, qui est l'évaluation des sollicitations dans les structures en interaction avec le sol:

- vingt-cinq années d'expérience ont permis aux utilisateurs de définir, petit à petit, des ordres de grandeur qui sont maintenant validés, non seulement par l'usage, mais encore par de nombreuses mesures sur ouvrages réels, du moins dans le domaine des soutènements [10, 11].

Toutefois, cette approche purement empirique débouche à theure actuelle sur un résultat paradoxal, ce qui explique peut-ètre que certains préfèrent encore s'en tenir à des méthodes traditionnelles de détermination du coefficient $k$, mème si elles sont en contradiction avec les résultats expérimentaux [7]; on constate en effet que :

- le coefficient de réaction du sol sollicité en butée par un ouvrage de soutènement est en moyenne trois fois supérieur [10] au coefficient de réaction déduit de la formule de Ménard et Bourdon [9], ce qui a jusqu'à présent été attribué à la non-linéarité du comportement du sol : le principal défaut des méthodes de calcul usuelles consiste en effet à schèmatiser le comportement du sol à l'état limite de service, donc dans un domaine de petites déformations, en utilisant des paramètres destinés à englober un domaine de déformations beaucoup plus vaste, et abusivement décrit par un modèle de cormportement élastique et linéaire :

- la formule de Ménard et Bourdon, applicable aux écrans de soutènement, est elle-même déduite de la formule établie par Ménard [8] pour évaluer le tassement des semelles de fondation:

- or cette dernière formule a elle-même été établie sur des bases expérimentales, et intègre donc déjà, du moins en partie, l'effet de la non-linéarité du sol.

Faut-il en conclure que la théorie de l'élasticité sousestime le déplacement réel des soutènements dans un rapport de l'ordre, non pas de 1 à 3 , mais peut-être de 1 à 10, ce qui parait peu plausible, ou bien que le mode de calcul des soutènements préconisé par Ménard n'est pas homogène avec celui des fondations ?

Quel que soit l'avenir des calculs aux ccefficients de réaction, ce paradoxe méritait d'être analysé, et le présent article propose donc une comparaison systématique entre l'approche suivi par Ménard et la théorie de l'élasticité linéaire, afin de quantifier les écarts de principe entre ces deux méthodes, ainsi qu'une analyse des différences entre les ordres de grandeur des coefficients de réaction applicables aux fondations superficielles et aux soutènements.
On constate ainsi, non seulement que le paradoxe précédemment signalé s'explique en réalité de façon logique, mais encore que l'approche théorique permet, contre toute attente, de retrouver des résultats qui avaient précédemment été établis sur une base exclusivement empirique, preuve que la méthode din cofficient de réaction, bien que très approximative, possède une cohérence interne insoupçonnée !

Ceci justifie a posteriori l'usage intensif qui en a été fait pour dimensionner la plupart des grands projets des trois dernières décennies, et débouche sur une formulation simple, générale, et compatible avec la plupart des résultats théoriques et expérimentaux aujourd'hui disponibles.

\section{2}

\section{Étude comparative des théories de Ménard et de l'élasticité linéaire pour le calcul du tassement des fondations superficielles}

La théorie de Ménard pour le calcul du tassement des fondations superficielles repose sur la distinction entre les parties isotrope et déviatoire des champs de contraintes et de déformations, donnant lieu à des coefficients de réaction respectivement égaux à $k$ et $k_{\mathrm{o}}$, le coefficient de réaction global s'exprimant alors sous la forme :

$$
1 / \mathrm{k}=1 / \mathrm{k}_{\mathrm{i}}+1 / \mathrm{k}_{\mathrm{d}}
$$

La partie isotrope clu tassement est assimilée à la diminution du rayon de la demi-sphère, ou du demi-cylindre de sol situé sous la fondation, tandis que la partie déviatoire est assimilée au tassement du reste du massif sol.

\section{1}

\section{Cas d'une fondation circulaire}

Dans ce cas, Cassan [2] a pu reconstituer le calcul de Ménard en supposant que la demi-sp̣hère de sol limitée par la fondation est soumise à une contrainte normale uniforme.

La partie isotrope du tassement se calcule alors par application directe du module d'élasticité isotrope, soit:

$$
k_{1}=E / i:(1-2 v)
$$

oủ $E$ est le module d'Young et $v$ le coefficient de Poisson du sol.

La partie déviatoire est décrite par la formule établie par Josselin de Jong [6] pour évaluer le tassement d'une sphère rigide, soit :

$$
\begin{aligned}
& k_{\mathrm{a}}=3 \mathrm{E} / \mathrm{r} \cdot(1+v) \\
& \mathrm{k}=3 \mathrm{E} / \mathrm{r} \cdot(4-5 \mathrm{v})
\end{aligned}
$$

Ces formules approchées sont à comparer à celles, rigoureuses, établies par Cordary et al. [3] pour calculer le tassement au centre d'une fondation circulaire souple, soit:

et:

$$
k_{i}=1,5 \cdot E / r \cdot(1+v) \cdot(1-2 v)
$$

$$
\left(\mathrm{K}_{\mathrm{d}}=1,5 \cdot \mathrm{E} / \mathrm{r} \cdot(1+\mathrm{v}) \cdot(2-\mathrm{v})\right.
$$

La théorie de l'élasticité conduit ainsi, dans le cas du tassement au centre d'une fondation circulaire, à l'expression: 


$$
k=E / 2 r \cdot(1+v) \cdot(1-v)
$$

Le rapport entre les coefficients de réaction respectivement déduits des calculs de Cassan et de Cordary est égal à 6. $(1+v) .(1-v) /(4-5 v)$, c'est-à-dire que les hypothèses simplificatrices faites par Ménard conduisent à surévaluer $\mathrm{k}$ dans un rapport compris entre 2,3 pour $\mathrm{v}=1 / 3$ et 3 pour $\mathrm{v}=1 / 2$.

Si l'on ne considère que la partie isotrope du tassement, ce rapport vaut $(1+v) / 1,5$, soit 0,9 pour $v=1 / 3$ à 1 pour $\mathrm{v}=1 / 2$

C'est donc exclusivement de la partie déviatoire que provient la surévaluation, écale à $2 .(2-v)$, soit 3,3 pour $v=1 / 3$ à 3 pour $v=1 / 2$

A ce stade, il apparait donc une surévaluation de $k$ dans un rapport de l'ordre de 2,3 à 3 , provenant essentiellement de l'assimilation du tassement déviatoire à celui provoqué par une sphère rigide soumise à une translation verticale.

On peut y voir la conséquence de deux facteurs - le champ généré par la translation verticale d'une sphère rigide n'est pas déviatoire;

- les deux champs ainsi superposés ne sont pas compatibles, puisque le domaine sphérique est supposé soumis à l'action d'une pression uniforme, tandis que les contraintes exercées par la sphère translatée sur le sol avoisinant ne sont nullement uniformes.

Une autre approche consisterait à associer tout simplement le champ déviatoire à l'expansion d'une cavité sphérique, ce qui revient à écrire :

d'oủ :

$$
k_{\mathrm{d}}=2 \mathrm{E} / \mathrm{r} \cdot(1+\mathrm{v})
$$

$$
k=2 E / 3 r \cdot(1-v)
$$

La figure 1 montre que cette schématisation, si elle présente l'avantage de respecter la condition de continuité des contraintes, demeure très approximative, puisqu'elle ne vérifie pas plus que la précédente, la condition de compatibilité des déformations.

La surévaluation de $k$ qui en résulte est toutefois limitée à 4/3.(1+v), soit 1,8 à 2 .

On remarque que ce rapport n'est pas très différent de celui qui existe entre le tassement sous le centre et le tassement sous le bord d'une fondation circulaire souple [5], soit :

- tassement sous le centre : $y=2 \mathrm{pr} \cdot\left(1-\mathrm{v}^{2}\right) / \mathrm{E}$

- tassement sous le bord : $y=4 p r / \pi .\left(1-v^{2}\right) / E$

- rapport des tassements : $\pi / 2=1,6$.

Simulation du champ isotrope Simulation du champ déviatolre (compression d'une demi-sphère) (expansion d'une demi-sphère)
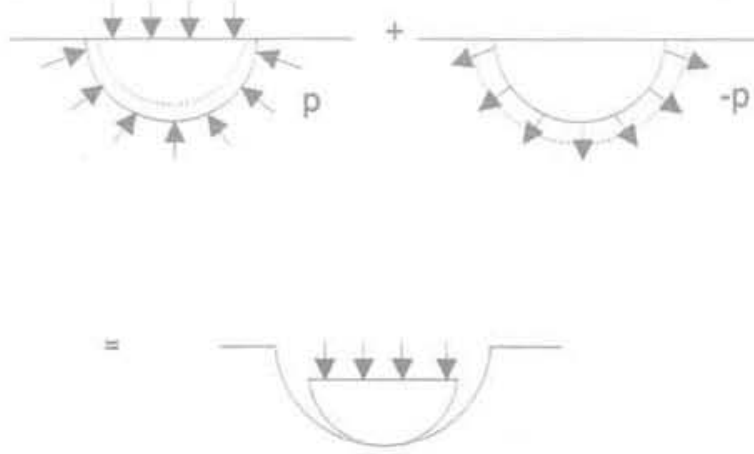

FiG. Décomposition schématique du tassement d'une fondation superficielle.

Schematic settlements under a shallow foundation.
On peut donc conclure que, dans son principe, la décomposition « géométrique » des champs isotrope et déviatoire imaginée par Ménard ne minore que de 10 à $30 \%$ le tassement sous le bord d'une fondation circulaire souple.

\section{0}

\section{Cas d'une semelle filante}

Dans le cas d'une semelle filante, Ménard introduit des coefficients de forme respectivement égaux à 1,5 pour la partie isotrope du tassement et 2,7 pour la partie dêviatoire, ce qui conduit aux formulations suivantes :

$$
\begin{aligned}
& \mathrm{k}_{\mathrm{i}}=\mathrm{E} / 1,5 \cdot \mathrm{r} \cdot(1-2 \mathrm{v}) \\
& \mathrm{k}_{\mathrm{d}}=3 \mathrm{E} / 2,7 \cdot \mathrm{r} \cdot(1+\mathrm{v}) \\
& \mathrm{k}=\mathrm{E} / \mathrm{r} \cdot[1,5 \cdot(1-2 v)+0,9 \cdot(1+v)]=\mathrm{E} / \mathrm{r} \cdot(2,4-2,1 v) .
\end{aligned}
$$

La théorie de l'élasticité, quant à elle, concluit à $\mathrm{k}=0$, puisque le tassement d'une semelle reposant sur un massif indéfini tend vers l'infini quand sa longueur tend elle-même vers l'infini.

Dans la pratique, on se console de ce résultat inexploitable en faisant valoir qu' une couche de sol homogène d'épaisseur infinie n'existe pas, et en définissant une semelle filante comme une fondation dont la longueur est grande vis-à-vis de la couche de sol compressible, ce qui débouche en pratique sur l'hypothèse des déformations planes, consistant à supposer nulles les déformations longitudinales.

Cette simulation de l'infini présente l'avantage pratique de ramener le tassement calculé à une valeur finie, mais ne donne malheureusement pas lieu à une formulation analytique.

Il est cependant possible d'en établir une expression approchée en décomposant le tassement, conformément à l'idée de Ménard, en une composante isotrope assimilée à la compression uniforme d'un demicylindre de sol, et une composante déviatoire assimilée à l'expansion de ce même demi-cylindre dans le massif de sol avoisinant.

Cette schématisation, bien que qrossière, est malgré tout justifiée par la validation qui en a précédemment était faite dans le cas d'une fondation circulaire.

Dans ce cas, le terme déviatoire se déduit directement de la théorie de l'expansion des cavités cylindriques, soit:

$$
k_{d}=E / r \cdot(1+v)
$$

Le terme isotrope, quant à lui, se décluit de l'expression de la loi de Hooke en coordonnées cylindriques, dans laquelle on écrit :

- l'égalité des déformations radiale et orthoradiale, soit $\varepsilon_{r}=\varepsilon_{\theta^{\prime}}$ compte tenu de I'hypothèse d'un champ de contraintes et de déformations isotrope dans le plan transversal ;

- la nullité des déformations longitudinales, soit $\varepsilon_{1}=0$, compte tenu de I'hypothèse des déformations planes.

En écrivant, par ailleurs :

et:

$$
\begin{aligned}
& \sigma_{\mathrm{f}}=\sigma_{\theta}=p \\
& \varepsilon_{\mathrm{r}}=\varepsilon_{\mathrm{\theta}}=y / \mathrm{r}
\end{aligned}
$$

on obtient :

$$
k=p / y=E / r \cdot(1+v) \cdot(1-2 v)
$$

soit, finalement:

$$
k=E / 2 r \cdot(1+v) \cdot(1-v)
$$


Il se trouve que cette expression est la même que celle du tassement sous le centre d'une fondation circulaire souple donnée par la théorie de l'élasticité.

Le rapport entre ce coefficient, qui résulte d'une approximation de la théorie de l'élasticité en déformations planes, et celui déduit des coefficients de forme de Ménard, est égal à :

$$
(2,4-2,1 v) / 2 \cdot(1+v) \cdot(1-v)
$$

qui vaut 1 pour $v=1 / 3$ et 0,9 pour $v=1 / 2$.

On constate donc, à ce stade, une excellente concordance entre les deux approches, ce qui est normal dans la mesure où elles reposent sur la mème schématisation : le coefficient de forme de 2,7 permet en fait de compenser l'écart initial dû à l'assimilation du tassement déviatoire d'une fondation circulaire à la translation verticale d'une sphère rigide.

Il apparait donc que la théorie de Ménard débouche en pratique, dans le cas d'une semelle filante, sur la formulation approchée :

$$
\mathrm{k}=\mathrm{E} / \mathrm{b} \cdot\left(1-\mathrm{v}^{2}\right)
$$

où b est la largeur de la fondation.

On vérifie toutefois, à partir de calculs plus rigoureux effectués à l'aide du programme aux éléments finis CESAR (Fig. 2), que les valeurs déduites de cette dernière expression s'apparentent plutôt au tassement sous le bord de la fondation $(9 \mathrm{~cm}$ à comparer à 11,5 pour $v=1 / 3$, et $7,5 \mathrm{~cm}$ à comparer à 7,5 pour $v=1 / 2)$, comme on l'avait déjà constaté dans le cas d'une fondation circulaire.
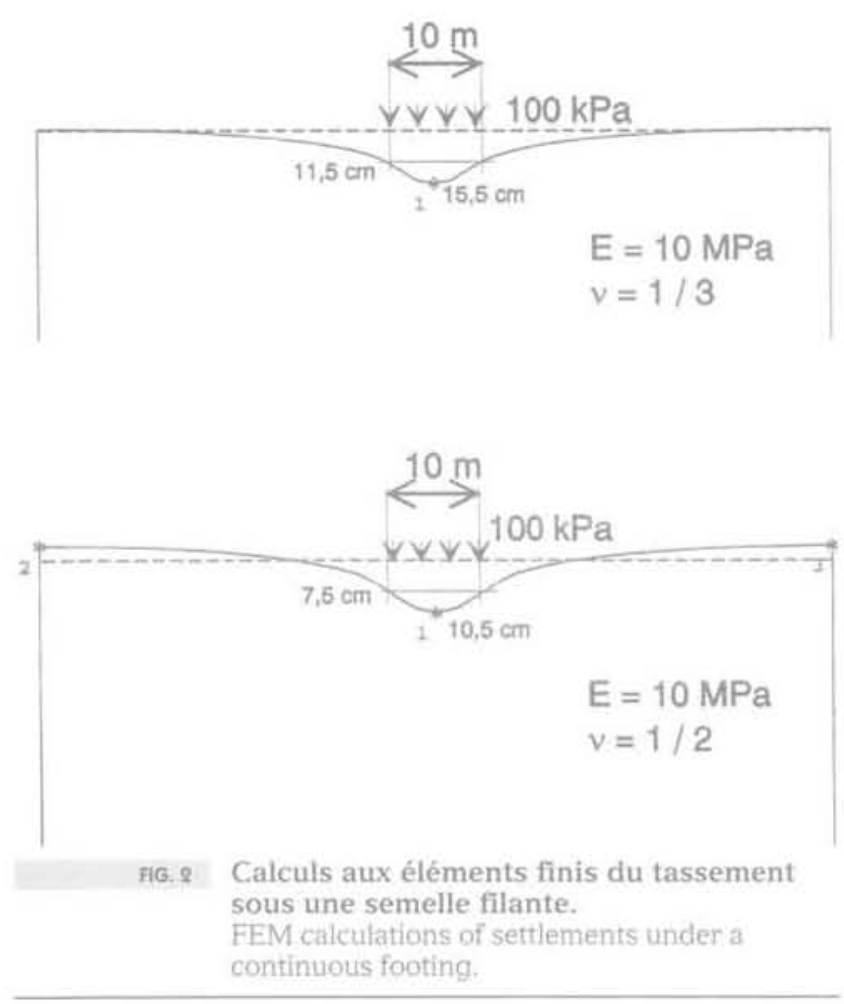

Une correction de la formule précédente peut ètre tentée à partir des formules analytiques rigoureuses rappelées par Giroud [5] dans le cas des fondations circulaires :

- tassement sous le centre : $y=2 p r .\left(1-v^{2}\right) E$

- tassement sous le bord : $y=4 p r / \pi \cdot\left(1-v^{2}\right) / E$

- tassement moyen : $y=16 \mathrm{pr} / 3 \pi \cdot\left(1-v^{2}\right) / \mathrm{E}$.
On vérifie que le rapport du tassement sous le centre au tassement sous le bord, égal à $\pi / 2$, n'est pas très différent de celui, inférieur d'environ $15 \%$, donné par les calculs aux éléments finis dans le cas d'une fondation de grande longueur.

On peut donc, avec un degré d'approximation raisonnable, déduire la valeur du coefficient de réaction "moyen $»$ de celle du coefficient de réaction sous le bord à partir d'un rapport identique pour les fondations de grande longueur et circulaires, soit 3/4.

En définitive, une expression approchée du cœefficient de réaction " moyen » du sol sous une fondation de grande longueur, qui correspond bien à la notion usuelle de ccefficient de réaction, est :

$$
\mathrm{k}=0,75 \cdot \mathrm{E} / \mathrm{b} \cdot\left(1-\mathrm{v}^{2}\right)
$$

Cette expression conduit à des tassements voisins de ceux directement déduits de la théorie de l'élasticité linéaire, et supérieurs à ceux déduits de la théorie de Ménard dans un rapport n'excédant pas 1,4.

Si l'on rappelle que la théorie de Winckler consiste à assimiler le sol à une infinité de ressorts de module $\mathrm{E}$ et de longueur 1 , soit $\mathrm{k}=\mathrm{E} / \mathrm{l}$, on peut encore dire que la longueur des « ressorts de sol » équivalents à la théorie de l'élasticité linéaire du point de vue du tassement moyen sous une fondation de grande longueur est:

$$
\mathrm{I}=\mathrm{b} \cdot\left(1-\mathrm{v}^{2}\right) / 0,75
$$

ou encore 1,2 .b pour $v=1 / 3$ à b pour $v=1 / 2$.

Autant dire qu'en pratique, on ne se trompe pas beaucoup en assimilant le sol à une juxtaposition de ressorts de module d'élasticité égal à celui du sol et de longueur égale à 1,1 fois la largeur de la fondation.

Pour fixer les idées, cette approximation conduit, dans le cas d'une fondation de $10 \mathrm{~m}$ de largeur, reposant sur un sol de module d'élasticité égal à $10 \mathrm{Mpa}$ et soumis à une surcharge de $100 \mathrm{kPa}$, à un tassement moyen de $11 \mathrm{~cm}$, alors que les calculs aux éléments finis indiquent un tassement moyen variable de $13 \mathrm{~cm}$ pour $v=1 / 3$ à $9 \mathrm{~cm}$ pour $\mathrm{v}=1 / 2$.

\section{3}

\section{Introduction du module pressiométrique}

Les développements précédents supposent le comportement du sol élastique, linéaire, homogène et isotrope, et ne peuvent donc prétendre à une simulation parfaite de la réalité, qui est évidemment beaucoup plus complexe.

On conçoit par conséquent que Ménard ait jugé nécessaire d'introduire dans ses formulations un cœefficient d'ajustement empirique, appelé u coefficient de structure $x$ et désigné par $\alpha$, et de distinguer le module pressiométrique, associé à un mode particulier de chargement du sol, ainsi qu'à un inévitable remaniement, du module d'élasticité de la théorie.

Le cơfficient $\alpha$ étant initialement défini comme le rapport entre le module pressiométrique et le module d'élasticité du sol dans un charnp de contraintes isotrope, l'expression du ccefficient de réaction isotrope devient, dans le cas d'une fondation de grande longueur:

$$
\mathrm{k}_{\mathrm{i}}=\mathrm{E}_{\mathrm{M}} / \alpha \cdot 1,5 \cdot \mathrm{r} \cdot(1-2 \mathrm{v})
$$

En pratique, Ménard introduit à ce stade un cœefficient multiplicateur de 1,5 qui annule l'effet du cœefficient de forme de 1,5 et conduit finalement à l'expression : 


$$
k_{i}=E_{M} / \alpha \cdot r \cdot(1-2 v)
$$

Dans le champ déviatoire, Ménard ne remet pas en cause la représentativité de $\mathrm{E}_{\mathrm{W}}$ dans la mesure où le module pressiométrique est lui-méme mesuré dans un champ déviatoire.

Ayant cependant constaté que le tassement déviatoire n'est pas aussi proportionnel à la largeur de la fondation que le voudrait la théorie de l'élasticité linéaire, il remplace l'expression:

par :

$$
\mathrm{k}_{\mathrm{d}}=3 \mathrm{E}_{\mathrm{M}} / 2,7 \cdot \mathrm{r} \cdot(1+\mathrm{v})
$$

avec : $r_{0}=0,30 \mathrm{~m}$.

$$
k_{\mathrm{d}}=3 \mathrm{E}_{\mathrm{N}} / \mathrm{r}_{0} \cdot\left(2,7 . \mathrm{r} / \mathrm{r}_{0}\right)^{\alpha} \cdot(1+v)
$$

On en arrive ainsi, dans le cas où $v=1 / 3$ à l'expression bien connue :

$$
\mathrm{k}=\mathrm{E}_{\mathrm{M}} /\left(\alpha \cdot \mathrm{b} / 6+0,13 \cdot(4,5 \cdot \mathrm{b})^{\mathrm{a}}\right)
$$

Il est intéressant, indépendamment de toute considération théorique, d'évaluer l'écart entre cette formulation empirique et la formulation plus théorique du paragraphe précédent.

Pour ce faire, on tiendra compte des recommandations de la Société internationale de mécanique des sols et travaux de fondations [1], qui assimilent le rapport $E_{\mathrm{V}} / \alpha$ non plus au module d'Young dans un champ isotrope, mais au module œdométrique, soit :

$$
E_{M} / \alpha=E \cdot(1-v) /(1+v) \cdot(1-2 v)=1,5 \cdot E \text { pour } v=1 / 3
$$

Compte tenu de tout ce qui précède, le rapport entre le coefficient de réaction de Ménard et celui déduit de la théorie de l'élasticité linéaire est égal à :

$$
\begin{gathered}
\text { b. }\left(1-v^{2}\right) / 0,75 \cdot E \cdot 1,5 \cdot \alpha \cdot E /\left(\alpha \cdot b / 6+0,13 \cdot(4,5 \cdot b)^{\alpha}\right)= \\
2 \cdot \alpha \cdot\left(1-v^{2}\right) \cdot b /\left(\alpha \cdot b / 6+0,13 \cdot(4,5 \cdot b)^{\alpha}\right)
\end{gathered}
$$

Dans le cas d'un sable, pour lequel on peut, en première approximation, admettre que $\mathrm{v}=\alpha=1 / 3$, ce rapport varie de 2,2 pour $b=1 \mathrm{~m}$ à 5,8 pour $\mathrm{b}=10 \mathrm{~m}$.

Dans le cas d'une argile saturée, ce rapport ne peut être évalué, puisque le module cedométrique d'un sol non drainé est théoriquement infini : il est probable que l'assimilation du module pressiométrique au module œdométrique n'est pas justifiée dans ce cas, ou plus exactement que le module pressiométrique ne caractérise que le comportement drainé du sol, puisqu'il est conventionnellement défini à partir du module déviatoire en supposant $v=1 / 3$.

Si $v=1 / 3$ et $\alpha=1 / 2$, on obtient un rapport variable de 2,5 à 5,2 , peu différent des valeurs précédentes.

On retiendra donc que le coefficient de réaction de Ménard est notablement plus raide que celui déduit de la théorie de l'élasticité, qu'il surestime clans un rapport de l'ordre de 2 à 6 . Dans la mesure où les deux approches s'appliquent en l'occurrence à des terrains réputés homogènes, il faut probablement y voir, essentiellement, une conséquence de la non-linéarité du comportement du sol, qui constitue une propriété incontestable.

A noter que, si l'on maintient la relation $E_{M} / \alpha=E$ initialement proposée par Ménard au lieu de la relation $\mathrm{E}_{\mathrm{M}} / \alpha=1,5$. E proposée par la SIMSTF, le rapport entre les coefficients déduits des deux approches devient 1,5 fois plus faible, c'est-à-dire en pratique variable de 1,5 à 4 seulement.

\section{Coffficient de réaction du sol vis-à-vis des tunnels}

L'approche la plus utilisée en France consiste, dans le cas où les variations de contraintes exercées par le sol sur le tunnel peuvent être supposées uniformes (cas d'un collecteur souple soumis à une pression intérieure hydrostatique par exemple), à utiliser le résultat donné par la théorie de l'élasticité linéaire dans le cas d'un champ déviatoire, soit :

$$
k=E / r \cdot(1+v)
$$

Il est remarquable que ce résultat ne s'applique, en toute rigueur, qu'au cas particulier d'une action uniforme du sol, associée à une simple dilatation fou à une simple contraction) du tunnel, alors que la théorie du coefficient de réaction a pour principal objectif l'évaluation des moments fléchissants associés à des chargements non uniformes...

L'expression précédente peut, par ailleurs, ètre comparée à la formulation empirique proposée par Ménard pour le terme déviatoire, soit :

$$
\mathrm{k}=3 \mathrm{E}_{\mathrm{M}} / \mathrm{r}_{0} \cdot\left(2,7 \cdot \mathrm{r} / \mathrm{r}_{0}\right)^{0} \cdot(1+\mathrm{v})
$$

ou encore, compte tenu de la relation approchée $E_{M} / \alpha=1,5$. E, et dans le cas envisagé précédemment où $\mathrm{v}=\alpha=1 / 3$ à :

$$
\mathrm{k}=3,75 \cdot \mathrm{E} /(\mathrm{g} \cdot \mathrm{r})^{1 / 3}
$$

Le rapport entre les valeurs déduites des deux expressions précédentes est égal à $5 . r /(9 . r)^{1 / 3}$, et varie donc de 1,5 pour $r=0,5 \mathrm{~m}$ à 7 pour $\mathrm{r}=5 \mathrm{~m}$.

Le coefficient de Ménard surestime donc celui déduit de la théorie de l'élasticité, dans le cas des tunnels sollicités uniformément, dans un rapport de l'ordre de 1,5 à 7 , d'autant plus élevé que le rayon du tunnel est plus grand, à comparer au rapport de l'ordre de 2 à 6 trouvé dans le cas des fondations superficielles.

Là encore, cet écart est probablement imputable à la non-linéarité du comportement du sol, bien évidemment ignorée par la théorie de l'élasticité linéaire.

A noter toutefois que, contrairement au cas des fondations superficielles, la pratique retient la valeur de $k$ déduite de la théorie de l'élasticité et non celle déciuite de la théorie de Ménard, probablement par manque de support expérimental direct, ce qui conduit paradoxalement à des valeurs de $k$ inférieures pour les tunnels alors que la disparition des déformations isotropes justifierait théoriquement un ccefficient plus élevé.

Le problème est qu'aucune des théories existantes ne traite le cas le plus intéressant en pratique, qui est celui des chargements non uniformes susceptibles de solliciter le revêtement en flexion.

\section{4}

\section{Coefficient de réaction du sol vis-à-vis des écrans de soutènement}

\section{1}

\section{Théorie de Ménard et Bourdon}

Le cœefficient de réaction élastique du sol en butée sous le niveau du fond de fouille est classiquement éva- 
Iué à partir de la formule proposée par Ménard et Bourdon, dont le principe consiste à assimiler la fiche, $\mathrm{f}$, à une semelle verticale de largeur $\mathrm{a}=2 / 3, \mathrm{f}$ :

$$
\mathrm{k}=\mathrm{E}_{\mathrm{M}} /\left(\alpha \cdot \mathrm{a} / 2+0,13 \cdot(9 \cdot \mathrm{a})^{\alpha}\right)
$$

Cette formulation suppose, comme l'avait d'ailleurs fait auparavant Terzaghi [12], que le déplacement de l'écran, sous l'effet d'une pression horizontale p appliquée sur une hauteur a au-dessous du fond de foulle. est égal à la somme des déplacements qui seraient obtenus sous l'effet d'une pression horizontale p appliquée sur une hauteur 2,a sur un massif de sol non excavé, ce qui annule le déplacement vertical au niveau du fond de fouille, et d'un déchargement, libérant le degré de liberté correspondant au soulèvement du sol en fond de fouille.

La prise en compte du chargement sur une hauteur égale à 2.a se traduit par l'assimilation de a à r au lieu de 2.r dans la formule de Ménard applicable aux semelles.

La prise en compte du déchargement au niveau du fond de fouille se traduit forfaitairement par la division par 1,5 du coefficient de réaction isotrope.

On notera que ce rapport est égal au rapport entre module cedométrique et module d'Young pour $\mathrm{v}=1 / 3$, et qu'il s'agit donc d'une prise en compte par excès de la décompression du sol, puisque les déformations verticales en l'absence d'excavation ne sont pas nulles comme dans un champ œdométrique, et que le module d'élasticité apparent avant déchargement est donc nécessaire inférieur au module cedométrique.

En dépit de ce coefficient dont la valeur est approximative, le raisonnement lui-même, dont les étapes sont décomposées sur la figure 3 , est rigoureux, et la formule de Mẻnard et Bourdon pour les soutènement peut donc être a priori considérée comme cohérente avec la formule de Ménard pour les semelles filantes, à l'anisotropie du sol près.

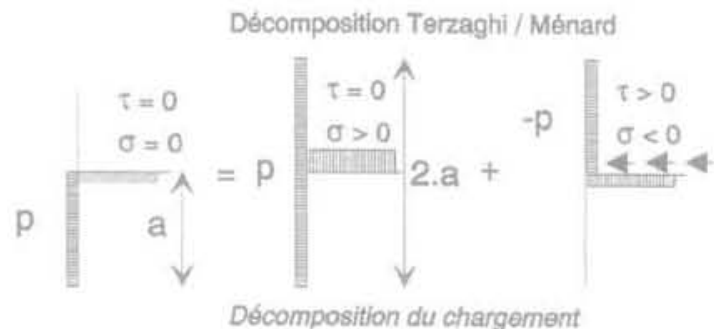

Décomposition du chargement

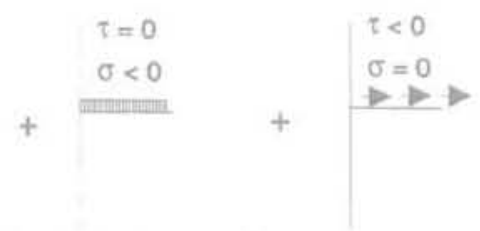

Annulation des contraintes au niveau du fond de foulle

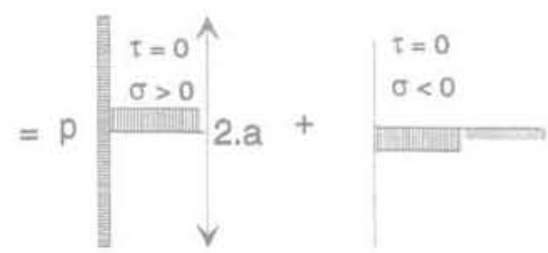

FiG.3 Décomposition schématique des contraintes appliquées sur la fiche d'un écran.

Schematic action of the embeded part of a retaining wall.
Les valeurs déduites de cette formule sont notablement plus faibles que celles applicables aux semelles: ainsi, lorsque $\alpha=1 / 3$, on trouve un rapport variable de 1,6 pour $\mathrm{a}=1 \mathrm{~m}$ à 2,2 pour $\mathrm{a}=10 \mathrm{~m}$, soit en pratique de l'ordre de 2.

Pour $\alpha=1 / 2$, on trouve de méme un rapport variable de 1,8 à 2,2 , donc également de l'ordre de 2 .

Cet ordre de grandeur est vraisemblable si l'on adimet que l'excavation a pour effet, non pas tant de décomprimer le terrain sous le fond de foullle, que d'annuler les contraintes de cisaillement, et donc de concentrer sous le fond de fouille les contraintes horizontales qui auraient été diffusées de part et d'autre en l'absence d'excavation: cette concentration des contraintes, et par conséquent des déformations, qui leur sont proportionnelles, justifie donc bien un coefficient réducteur de l'ordre de 2 vis-à-vis d'un chargement horizontal appliqué au voisinage du niveau de l'excavation.

\section{2}

\section{Comparaison entre théorie élastique, théorie pressiométrique et résultats expérimentaux}

On a pu vérifier [11] qu'en pratique, pour les valeurs de a supérieures à $2 \mathrm{~m}$, la formule de Ménard et Bourdon est pratiquement équivalente à :

$$
k=1,2 . E_{M} / \alpha . a
$$

ou encore, compte tenu de l'approximation $\mathrm{E}_{\mathrm{M}} / \alpha=$ 1,5. E (assimilation de $E_{N} / \alpha$ au module cedométrique, et comportement drainé du sol forfaitairement caractérisé par $\mathrm{v}=1 / 3$ ) :

$$
\mathrm{k}=1,8, \mathrm{E} / \mathrm{a}
$$

On a vu par ailleurs que la formule de Ménard pour les fondations, dont dérive la formule de Ménard et Bourdon pour les écrans, surévalue le ccefficient de réaction déduit de la théorie de l'élasticité dans un rapport de l'ordre de 2 à 6 pour les valeurs du paramètre dimensionnel comprises entre 1 et $10 \mathrm{~m}$, soit une valeur moyerne de l'ordre de 4 pour les valeurs courantes de a, comprises entre 2 et $10 \mathrm{~m}$.

Par conséquent, une formulation du coefficient de réaction homogène avec les résultats de la théorie de lélasticité serait, approximativement, $\mathrm{k}=(1,8 / 4=0,45)$. $E / a$, ce qui correspond à des « ressorts de sol » de longueur $1=a / 0,45$, approximativement double de a, ou encore deux fois plus souples que dans le cas des fondations superficielles (pour lesquelles on avait $1=1,1, b$ ), conformément à la théorie de Ménard et Bourdon.

Par ailleurs, l'expérience montre [10] que cette dernière théorie conduit à des valeurs en réalité sous-évaluées dans un rapport de l'ordre de 3, ce qui a jusqu'à présent été attribué à la non-linéarité du comportement de sol.

Il résulte de ce qui précède que la théorie de l'élasticité linéaire sous-estimerait, dans le cas des écrans, le coefficient de réaction déterminé expérimentalement dans un rapport de 1 à $3.4=12$ !

A ce stade, on dispose donc de différents résultats théoriques et expérimentaux dont les ordres de grandeur peuvent être résumés comme suit:

- l'ordre de grandeur $\mathrm{k}=\mathrm{E} /$ 2.a du cœefficient relatif aux soutènements est cohérent avec l'ordre de gran- 
deur $\mathrm{k}=\mathrm{E} / \mathrm{b}$ du coefficient relatif aux fondations superficielles, qui est lui-même cohérent avec les résultats issus de la théorie de l'élasticité linéaire;

- cependant, la formulation approchée $\mathrm{k}=\mathrm{E} / 2$ 2. a sousestime les valeurs obtenues expérimentalement dans un rapport supérieur à 10, ce qui ne peut être expliqué par le seul effet de la non-linéarité du sol.

\section{3}

\section{Comparaison entre les valeurs de $\mathrm{k}$ applicables aux écrans de soutènement et aux fondations superficielles}

L'explication de ce paradoxe se trouve obligatoirement dans le fait qu'on ne parle pas de la même chose, c'est-à-dire que les paramètres dimensionnels b, applicable aux fondations superficielles, et a, applicable aux êcrans de soutènement, ne sont pas assimilables.

Il n'est pas inutile de rappeler, à ce stade, que la définition du ccefficient $k$ résulte d'un choix arbitraire du dornaine sur lequel on définit le déplacement ("moyen $n$ auquel on s'intéresse.

En toute rigueur, la théorie de l'élasticité linéaire permet d'exprimer de façon intrinsèque (c'est-à-dire en fonction des seules propriétés du sol) le rapport F/y, où $\mathrm{F}$ est la force appliquée au terrain et y le déplacement en un point quelconque.

Dans la théorie approchée du coefficient de réaction, on s'intéresse au déplacement moyen dans un certain domaine, caractérisé par un paramètre dimensionnel d. unique dans le cas d'un problème à deux dimensions, à l'intérieur duquel on peut encore définir une pression moyenne $\mathrm{p}$, donc écrire $\mathrm{F}=\mathrm{p}$. $\mathrm{d}$, et définir un ccefficient $k=p / y=F / d . y$, cette fois-ci inversement proportionnel au paramètre $\mathrm{d}$.

Dans le cas des fondations superficielles, le domaine considéré est défini par $\mathrm{d}=\mathrm{b}$, où $\mathrm{b}$ est la largeur d'application de la charge.

On vérifie alors, comme on l'a vu précédemment en rappelant les résultats indiqués par Giroud [5], que ce domaine chargé est largement inférieur au domaine soumis à des déplacements significatifs, puisque le déplacement sous le bord est encore égal, dans le cas d'une fondation souple uniformément chargée, à $(2 / \pi=0,6)$ fois le déplacement maximal au centre.

Or, dans le cas des écrans de soutènement, le paramètre a définit, à la fois, le domaine d'application de la butée élastique et l'ensemble du domaine soumis à des đéplacements significatifs, puisque :

- Ménard et Bourdon [9], en écrivant $\mathrm{a}=2 / 3$. f, postulent l'existence d'un centre de rotation situé au voisinage du tiers inférieur de la fiche, et admettent donc implicitement une valeur du déplacement non significative à la profondeur a (ce que l'on vérifie bien en pratique) : - nous avons nous-mêmes [10, 11], en procédant à l'étude expérimentale conduisant à la formulation a = 1,7 . (EI,$\left.\alpha / E_{M}\right)^{1 / 3}$ dans le cas des écrans de longueur élastique inférieure à $2 / 3$. f, défini a comme étant la profondeur au-dessous de laquelle les déplacements mesurés deviennent inférieurs à $20 \%$, et non $60 \%$, du déplacement maximal, de façon à obtenir des résultats comparables avec la formule de Ménard et Bourdon.

On définit donc conventionnellement le ccefficient de réaction des écrans dans l'ensemble du domaine déformé, alors que, dans le cas des fondations superficielles, on ne s'intéressait qu'au domaine directement chargé par la fondation.

L'examen de la figure 2 montre que ce domaine directement chargé est environ deux fois plus petit que le domaine déformé, correspondant conventionnellement à des déplacements supérieur à $20 \%$ du déplacement maximal, compte tenu de la transmission latérale des efforts par cisaillement interne du sol.

Dans le cas d'une fondation souple de grandes dimensions chargée localement, on pourrait arbitrairement définir le coefficient de réaction dans le domaine correspondant à la zone chargée, comme précédemment, ou dans le domaine correspondant aux déformations significatives du sol, comme cela a été fait dans le cas des écrans.

Autrement dit, le coefficient de réaction est fonction, non seulement de la valeur du paramètre dimensionnel d, ce qui est connu, mais encore de la façon dont le paramètre d est défini, ce à quoi l'on prête généralement moins attention.

En pratique, on fait varier la définition de $\mathrm{d}$, de façon tout-à-fait légitime, en fonction du mode de sollicitation de l'ouvrage : ainsi, on adopte naturellement une valeur de d égale à la largeur b de l'ouvrage lorsque celui-ci charge le terrain de façon sensiblement uniforme (cas d'une semelle de faible largeur ou d'un collecteur souple uniformément rempli), mais on adopte une valeur a inférieure à la largeur totale de l'ouvrage lorsque celui-ci est chargé ponctuellement (partie supérieure d'un écran sollicitée par des appuis ponctuels (réactions de tirants ou butons), ou fiche d'un écran sollicitée par le torseur induit par la partie supérieure de cet écran).

Dans ce dernier cas, la valeur a est intermédiaire entre la fiche totale de l'ouvrage, soit $f$, et la profondeur b sur laquelle les déplacements varient peu (c'est-à-dire restent supérieurs à $60 \%$ de leur valeur maximale, par assimilation avec le comportement des fondations superficielles), comme cela apparait sur la figure 4.

Si l'on admet que b est environ deux fois plus petit que a, ce qui se vérifie à partir de la théorie des poutres sur appuis élastiques [4], dont les résultats sont indiquès sur le tableau I dans le cas d'une sollicitation ponctuelle, on peut alors décomposer le rapport de 1 à 12 signalé précédemment entre résultats théoriques et expérimentaux en:

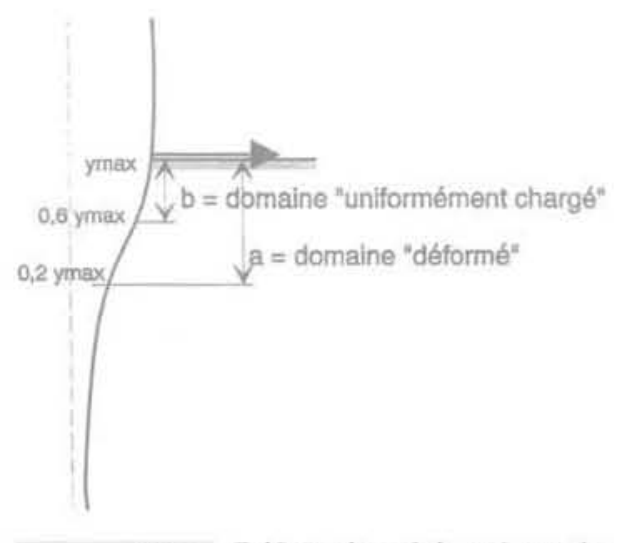

FG. 4 Déformée schématique de la fiche d'un écran.

Schematic behaviour of the embeded part of a retaining wall. 
- un écart, de l'ordre de 2, entre la théorie de Ménard et Bourdon et les résultats expérimentaux, dû au fait que cette théorie ne fait pas la distinction entre a et b; - un écart, de l'ordre de 6 , donc 1,5 fois plus grand que dans le cas des fondations superficielles, entre la théorie de l'élasticité linéaire et les résultats expérimentaux, essentiellement imputable à la non-linéarité du comportement du sol.

Il est toutefois important de noter que, si l'on adopte la relation originelle $E_{N} / \alpha=E$ au lieu de $E_{M} / \alpha=1,5$. $E$, l'écart entre résultats théoriques et experimentaux imputable à la seule non-linéarité du sol demeure voisin de 4, pour un écart global de l'ordre de 8.

Dans un souci de cohérence avec la théorie de Ménard, et compte tenu de ces ordres de grandeur plus vraisemblables, c'est cette relation originelle qui sera adoptée dans la suite de l'exposé, sachant que les résultats finals n'en sont pas affectés.

TAatEau- Déformée relative d'une poutre sur appuis élastiques.

Deflection of a beam resting on elastic supports

\begin{tabular}{l|l}
0 & 1 \\
\hline 0,05 & 0,9976 \\
\hline 0,1 & 0,9906 \\
\hline 0,15 & 0,9797 \\
\hline 0,2 & 0,9651 \\
\hline 0,25 & 0,9473 \\
\hline 0,3 & 0,9267 \\
\hline 0,35 & 0,9036 \\
\hline 0,4 & 0,8785 \\
\hline 0,45 & 0,8515 \\
\hline 0,5 & 0,8231 \\
\hline 0,55 & 0,7934 \\
\hline 0,6 & 0,7628 \\
\hline 0,65 & 0,7315 \\
\hline 0,7 & 0,6997 \\
\hline 0,75 & 0,6676 \\
\hline 0,8 & 0,6354 \\
\hline 0,85 & 0,6032 \\
\hline 0,9 & 0,5712 \\
\hline 0,95 & 0,5395 \\
\hline 1 & 0,5083 \\
\hline 1,05 & 0,4777 \\
\hline 1,1 & 0,4477 \\
\hline 1,15 & 0,4184 \\
\hline 1,2 & 0,3899 \\
\hline 1,25 & 0,3622 \\
\hline 1,3 & 0,3355 \\
\hline 1,35 & 0,3097 \\
\hline 1,4 & 0,2849 \\
\hline 1,45 & 0,2611 \\
\hline 1,5 & 0,2384 \\
\hline 1,55 & 0,2166 \\
\hline 1,6 & 0,1959 \\
\hline & \\
\hline 0
\end{tabular}

\section{4}

\section{Cœfficient de réaction du sol vis-à-vis des écrans souples sollicités localement}

Compte tenu de ce qui précède, la non-linéarité du comportement du sol revient grossièrement à le carac- tériser, dans le domaine des déformations usuelles, par un module d'élasticité $E_{s}=4 . E_{M} / \alpha$

Par ailleurs, le ccefficient de réaction du sol au voisinage de l'excavation peut être approximativement exprimé sous la forme:

$$
\mathrm{k}=\mathrm{E}_{s} /(2 \mathrm{~b})=\mathrm{E}_{\mathrm{s}} /(2, \mathrm{a} / 2)=\mathrm{E}_{\mathrm{s}} / \mathrm{a}=4 . \mathrm{E}_{\mathrm{M}} /(\alpha \mathrm{a})
$$

La relation approchée $\mathrm{k}=4 . \mathrm{E}_{\mathrm{M}} /(\alpha \mathrm{a})$ est identique, à $11 \%$ près, à celle qui était directement issue des résultats expérimentaux [10], soit $\mathrm{k}=(3,1,2=3,6)$ $E_{M} /(\alpha a)$.

La valeur de a, qui constitue un paramètre aussi important que le module d'élasticité lui-même, peut être évaluêe à partir de la théorie des poutres sur appuis élastiques et des résultats indiqués dans le tableau I : on vérifie ainsi que, dans le cas d'une sollicitation ponctuelle, la valeur de a correspondant à $20 \%$ cie déplacement résiduel est égale à $1,6, I_{\gamma}$ où $t_{0}$ est la longueur élastique de l'écran, elle-mème égale à $(4 . \mathrm{EI} / \mathrm{k})^{1 / 4}$, en désignant par El le produit d'inertie de l'écran.

Compte tenu de l'expression de $k$ donnée ci-dessus, on a, en définitive :

$$
\mathrm{a}=1,6 \cdot\left(4 \cdot \mathrm{EV} /\left(4, \mathrm{E}_{\mathrm{M}} /(\alpha \mathrm{a})\right)\right)^{2 / 4}
$$

soit :

ou encore :

$$
a^{3}=1,6^{4}, \text { EI }, \alpha, E_{M}
$$

$$
a=1,9 \cdot\left(E I, \alpha / E_{M}\right)^{1 / 3}
$$

Cette expression est à comparer à celle qui avait été précédemment trouvée, de façon purement empirique, à partir de l'examen d'une dizaine d'expérimentations sur ouvrages réels [11], soit :

$$
a=1,7 \cdot\left(E I, \alpha / E_{M}\right)^{13}
$$

qui correspondrait à a de l'ordre de 1,4. $I_{0}$.

La théorie et l'expérience conduisent donc en l'occurrence à des résultats dont l'écart n'excède pas $12 \%$, ce qui est remarquable, même s'il convient de ne pas oublier que le coefficient de réaction n'est rien d'autre qu'un paramètre artificiel dont la valeur ne pourra jamais être connue de façon parfaitement fiable à moins de $100 \%$ près.

On vérifie donc a posteriori la cohérence des différentes approches utilisées (théorie de l'élasticité linéaire, théorie des poutres sur appuis élastiques, et observations expérimentales), pour parvenir en fin de compte à une formulation homogène du coefficient de réaction, applicable à différents types d'ouvrages, à condition de tenir compte, dans chaque cas, d'une valeur du paramètre dimensionnel a ou b compatible avec le mode de chargement envisagé.

Rappelons les principaux ordres de grandeur mis en évidence au cours de la présente étude

- cas d'une semelle filante, avec un tassement sous le bord de l'ordre de $60 \%$ du tassement maximal : $\mathrm{k}=$ $\mathrm{E}_{\mathrm{s}} / \mathrm{b}$;

- cas d'un écran de soutènement, en définissant a comme le domaine des déplacements significatifs, ou encore supérieurs à $20 \%$ du dêplacement maximal : $\mathrm{k}=$ $\mathrm{E}_{3} / \mathrm{a}$, avec a de l'ordre de $1,5 \cdot \mathrm{I}_{0}$;

- non-linéarité du sol : $\mathrm{E}_{\mathrm{s}}=4 . \mathrm{E}_{\text {Young }}=4 . \mathrm{E}_{\mathrm{M}} / \alpha$ (domaine de validité : $\mathrm{a}=2$ à $10 \mathrm{~m}$ ).

On vérifie en particulier que ces ordres de grandeur sont compatibles avec les formulations directement déduites de l'observation du comportement des ouvrages [11], soit : 
$-a=1,7 \cdot\left(\mathrm{EI} \cdot \alpha / \mathrm{E}_{\mathrm{M}}\right)^{1 / 3}$

$-\mathrm{k}=2,1 .\left(\mathrm{E}_{\mathrm{M}} / \alpha\right)^{4 / 3} /(\mathrm{EI})^{5 / 3}$.

On notera que la formulation $k=E_{s} / a$ s'applique aussi bien à la fiche d'un écran qu'à sa partie supérieure, sollicitée en butée élastique par les appuis ponctuels que constiluent les tirants ou butons.

En effet, dans ce dernier cas, la multiplication par 2 du paramètre dimensionnel $b=a / 2$ demeure justifiée, non plus pour tenir compte de la présence de l'excavation, mais simplement par raison de symétrie (Fig. 5).

En fin de compte, on notera que la formule approchée $\mathrm{k}=\mathrm{E} / \mathrm{d}$ s'applique dans tous les cas, la valeur de $\mathrm{d}$ étant intermédiaire entre la dimension totale de l'ouvrage b, lorsque cette dimension est petite ou le chargement uniforme, et 1,5. I dans le cas d'ouvrages souples soumis à des chargements localisés.

Bien évidemment, si la prise en compte de $\mathrm{d}=1,5 . \mathrm{I}_{0}$ est justifiée dans la plupart des cas (notamment ceux des ouvrages réels ayant servi de support aux formulations empiriques $[10,11]$, des valeurs plus élevées peuvent être mieux adaptées dans certains cas : c'est ce qui a été récemment mis en évidence dans le cas du métro du Caire, avec une paroi moulée de $50 \mathrm{~m}$ de profondeur uniformément sollicitée par le rabattement effectué à l'intérieur de l'enceinte.

Toutefois, même lorsque le choix $d=b$ est mieux approprié du point de vue de la représentativité des déplacements, le choix $\mathrm{d}=1,5.1$ demeure généralement licite du point de vue de l'évaluation des efforts de flexion, qui résultent essentiellement de la partie non uniforme du chargement, comme l'avait d'ailleurs indiqué Westergaard [13] dans le cas des radiers.

On peut raisonnablement supposer qu'il en est de même, en particulier, dans le cas des tunnels.

On rappellera enfin que la théorie approchée du coefficient de réaction n'a jamais eu pour objet de calculer des déplacements, mais uniquement d'évaluer des sollicitations.

\section{5}

\section{Conclusion}

La comparaison systématique entre la théorie de Ménard et la théorie de l'élasticité a permis d'expliquer les écarts observés avec les résultats expérimentaux.

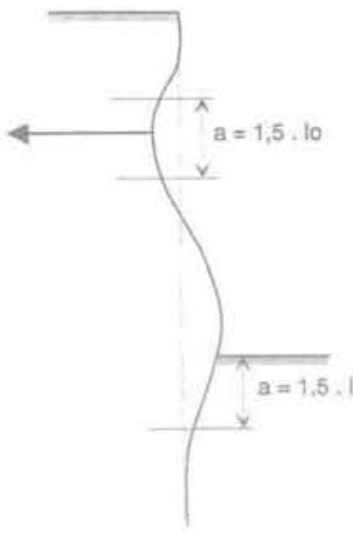

FG.5 Longueur caractéristique d'un écran souple.

Equivalent lenght of a flexible wall.

En particulier, il s'avère que la non-linéarité du comportement du sol est déjà en grande partie prise en compte dans la théorie de Ménard, et que les écarts observés dans le domaine des soutènements proviennent essentiellement du fait que la formule de Ménard et Bourdon assimile la fiche d'un écran à une semelle de fondation, sans tenir suffisamment compte des différences de comportement entre ces deux types d'ouvrage, dont les modes de sollicitation et de déformation sont relativement éloignés.

La théorie des poutres sur appuis élastiques a enfin permis de retrouver la valeur expérimentale du paramètre dimensionnel caractéristique des écrans souples, qui est de l'ordre de 1,5 fois la longueur élastique de l'écran pour les modes de sollicitations usuels.

L'ensemble des théories et expérimentations aujourd'hui disponibles permet en définitive de valider, dans le domaine d'utilisation qui lui est propre, la théorie approchée du coefficient de réaction et les méthodes de détermination mises au point par les entreprises spécialisées au cours des dernières décennies.

Le bien-fondé de cette approche empirique n'enlève rien à l'intérêt d'évoluer vers des méthodes numériques plus rigoureuses, permettant de prendre en compte le comportement non linéaire des terrains de façon plus satisfaisante qu'à partir de coefficients d'ajustement forfaitaires.

\section{Bibliographie}

[1]Amar S., Clarke B.G.F., Gambin M.P. Orr T.L.L. - Utilisation des résultats des essais pressiométriques pour le dimensionnement des fondations en Europe, 1 re partíe : pressiométre Ménard et pres. siomètre autoforeur. SIMSTF, Comite technique régional européen $n^{\circ} 4$, Pressiomètres, A.A. Balkema, 1991.

[2] Cassan M. - Les essais in situ en mécanique des sols. Paris, Evrolles 1978.

[3] Cordary D., Gambin MP., Van Wam. beke A. - " Prévision des tassements à l'aide du pressiometre n. X' CIMSTF. Stockholm, vol. 1, 1981, p. 95.

[4] Courbon J. - Résistance des matériaux. Paris Dunod. 1971

[5] Giroud J.P. - Tassement et stabilité des fondations superficielles, Presses universitaires de Grenoble, 1975 .

[6].Josselin de Jong G. - "Application des fonctions d'Airy aux problèmes de consolidation 1.. IVe CIMS, Londres. 1957, p. 320

[7]Londez M. Namur S.. Schmitt P. - "AnaIvse des mesures de déformation d'une parol moulée à Colombes », XIVe CIMSTF, Hambourg, vol. 2, 1997, p. 1323.

[8] Ménard L. - te Le tassement des fondations et les techniques pressiométriques. Bilan après dix ans de résultats expérimentaux n. Annales de lTTBTP. 1971.

[9] Menard L Bourdon C - Calcul des rideaux de soutènement. Méthode nouvelle pre- nant en compte les conditions réelles d'encastrement. Sols-Soils, n"12, 1965.

[10] Schmitt P. - uEtude experimentale de la sollicitation exercée par le sol sur les ouvrages de soutènement souples \%. Revue Francaise de Géotechnique, n"28, 1984.

[11] Schmitt P. - " Méthode empirique d'évaluation du coefficient de réaction du sol vis-â-vis des ouvrages de soutè. nement souples $¥$. Revue Française de Géotechnique, $\mathrm{n}^{\circ} 71,1995$.

[12] Terzaghi K. - Evaluation of coefficients of subgrade reaction. Geotechnique. vol.5, 1955, p. 297.

[13] Westergaard H.M. - -Stresses in concrete pavements computed by theoretical analysis $»$, Public Roads, $n^{\circ} 7,1926, \mathrm{p} .25$. 